\title{
Experimental Investigation of Pendant and Sessile Drops in Microgravity
}

\author{
Zhi-Qiang Zhu • David Brutin · Qiu-Sheng Liu • \\ Yang Wang • Alexandre Mourembles • \\ Jing-Chang Xie $\cdot$ Lounes Tadrist
}

Received: 30 November 2009 / Accepted: 25 June 2010 / Published online: 13 July 2010

(C) Springer Science+Business Media B.V. 2010

\begin{abstract}
The experiments regarding the contact angle behavior of pendant and sessile evaporating drops were carried out in microgravity environment. All the experiments were performed in the Drop Tower of Beijing, which could supply about $3.6 \mathrm{~s}$ of microgravity (free-fall) time. In the experiments, firstly, drops were injected to create before microgravity. The wettability at different surfaces, contact angles dependance on the surface temperature, contact angle variety in sessile and pendant drops were measured. Different influence of the surface temperature on the contact angle of the drops were found for different substrates. To verify the feasibility of drops creation in microgravity and obtain effective techniques for the forthcoming satellite experiments, we tried to inject liquid to create bigger drop as soon as the drop entering microgravity condition. The contact angle behaviors during injection in microgravity were also obtained.
\end{abstract}

Keywords Drop • Evaporation • Contact angle • Wettability $\cdot$ Drop tower

Z.-Q. Zhu · Q.-S. Liu ( $\bigotimes)$ · Y. Wang · J.-C. Xie

Key Laboratory of Microgravity (National Microgravity Laboratory), Institute of Mechanics, Chinese Academy of Sciences, Beijing 100190, China

e-mail: liu@imech.ac.cn

D. Brutin · A. Mourembles $\cdot$ L. Tadrist Laboratorie I.U.S.T.I., Ecole Polytechnique Universitaire de Marseille, Technopole de Chateau Gombert,

5 rue Enrico Fermi, 13453 Marseille, France

\section{Introduction}

Drop is an ordinary phenomenon in nature, which we are confronted with in everyday life. With the development of the industry, drop has attracted more and more scientific interests due to its broad applications in practical fields, for instance in thermal management and combustion systems. According to previous literatures, there are usually two research aspects (Picknett and Bexon 1977; Birdi et al. 1989; Law 1982; Sirignano 1983). Firstly, people made use of the evaporation and condensation of drops to achieve high heat transfer rates. Secondly, people explored the mechanisms of small drops injection in combustion systems. In present paper, we are focusing the evaporating drops.

Evaporation of drops is an efficient way to cool a heated surface by latent heat of vaporization when the drops change phase. And the small evaporating drops could provide greater heat transfer flux than thick liquid layers and traditional air cooling techniques. As a result, the study on cooling of evaporating drops has attract growing attention, motivated by the great application in nuclear thermal control, microelectronic industries and biology manufacture.

The mechanisms of evaporating process of drops are still absent of comprehensive understanding even if it has been widely used in industry to enhance heat transferring. A better understanding and clear knowledge of the physical mechanism could make the present thermal engines and microelectronic chips to be more powerful and minimal at the same time.

Evaporation could evidently change the temperature distribution along the fringe of the drop. The changing temperature profile synchronously change the evaporating rate, making the local evaporating rates very 
different at the surface of the drop. Based on previous research, the drop evaporation is enhanced in the vicinity of the triple line. Grandas (2004) found that the evaporation of a drop posed on a heated horizontal surface was strongly localized in the vicinity of triple line.

The triple line is usually reflected by the contact angle, which could be used to predict and analyze the drop evaporation. Ponter and Boyes (1972) firstly studied the contact angle of the sessile drop. They found that only small drops (smaller than $5 \mathrm{~mm}$ ) changed their contact angles under variable drop diameter. BourgesMonnier and Shanahan (1995) studied the influence of surface roughness on the contact angle and diameter of the drop. And two different evaporating manners were found depending on the surface roughness. They also proposed a model to calculate the coefficient of diffusion of the liquid vapor in air. Subsequently, Panwar et al. (2003), Grandas et al. (2005) and Takata et al. (2005) performed similar experiments with different surfaces. They all found that the contact angle changed depending on the physical properties of the surfaces. Crafton and Black (2003) studied the contact angle behavior with variable drop diameters, and measured the evaporating rates and heat fluxes for different liquid drops. They found that evaporating rate was not the main parameter to influence the heat flux. Shahidzadeh-Bonn et al. (2006) carried out experiments to study the evaporation of drops on a substrate which was wetting to the liquid. And they discussed the relation between the drop diameter and the time under different heat transfer modes.

Actually, besides surface roughness, conductivity, liquid characteristic and drop diameter, fluid motion in the liquid drop is a main effect to effect the heat transfer. Bigioni et al. (2006) found that the convection inside the drop made the particles easily to accumulate in the vicinity of the triple line. Ruiz and Black (2002), Savino et al. (2002) carried out numerical simulations to study thermocapillary convection in sessile drops and pendant evaporating drops, respectively. And they found different temperature distributions inside the drops comparing to the results that neglect fluid motion.

In above mentioned literatures, all the experimental and numerical research are carried out under normal gravity. Motivated by increasing applications of the evaporative drops in microgravity environment, such as heat pipes and heat transfer cells, there are growing experimental and numerical research on the heat transfer mechanism of drops (Brutin et al. 2009; Akira 2005). With the development of space industry, the mechanisms of evaporating drops in microgravity need full identification to enhance heat transfer rates in thermal management devices.

The mechanisms of evaporating drops in microgravity are complex and need a better understanding. In this paper, we will present the experimental results performed in the China drop tower concerning the contact angles in two kinds of evaporating drops, sessile and pendant drops.

In the following, we will first specify the details of the experimental apparatus and the measurement techniques in "Experimental Setup and Measurement Techniques". In "Results and Discussion", we will present and discuss our experimental results, including the wettability at different surfaces, contact angles dependence on the surface temperature, contact angle variety in sessile and pendant drops. Comparison will also be performed between the parabolic flight and Drop Tower results on contact angle depending of drop diameter. In addition, pendant drop contact angle behaviors during injection in microgravity were investigated with the goal of obtaining reference data for future satellite experiments. Finally, conclusions are stated "Conclusion".

\section{Experimental Setup and Measurement Techniques}

The overall schematic setup of the experimental apparatus for the investigation of contact angles in an evaporating drops is presented in Fig. 1. Two substrates (each $10 \mathrm{~mm}$ in diameter) are put into a test cell, where the sidewalls are made of plexiglass for optics observations.

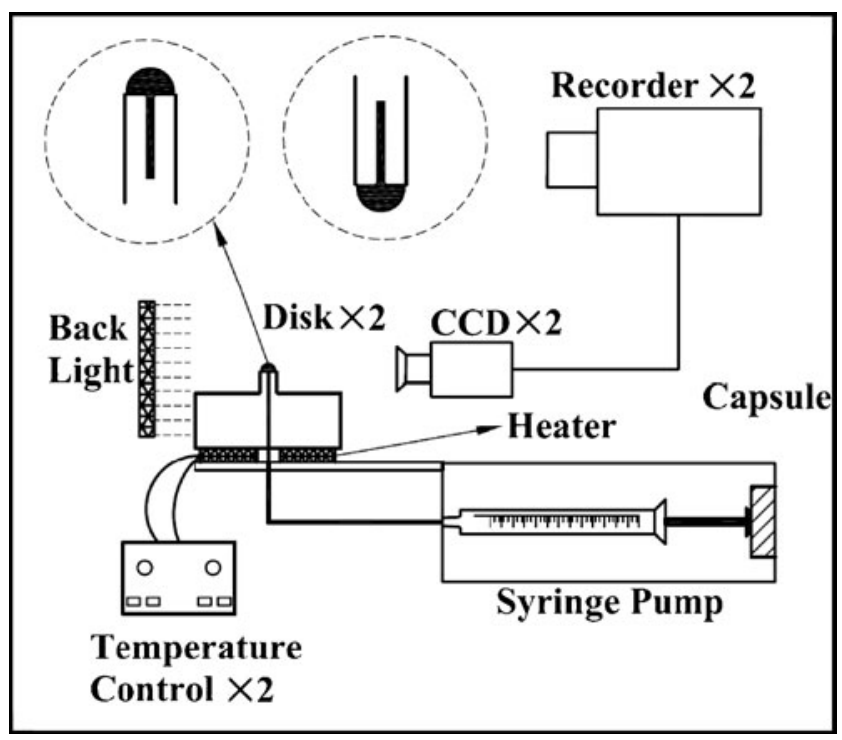

Fig. 1 Schematic drawing of the experimental apparatus 
The top of the test cell is a plexiglass for monitoring the drop injection process. In a word, the test cell is designed to play the role of insulation outer air permitting light through. The two substrates made of aluminum are used to create and sustain the evaporating drops. The thermal conductivity of the aluminum is large enough to ensure the uniform bottom temperatures of the drops. In our experiments, the two substrates were usually covered by two kinds of top surfaces, aluminum and polytetrafluoroethylene (PTFE), respectively, which were thin sheets with thickness of 200 $\mu \mathrm{m}$. Different surfaces were useful to analyze different wettability of drops. Note that drop shapes are very sensitive to contamination on the surfaces. Motivated by the request of performing all the experiments with same clean surface condition, prior to each experiment, the surfaces were sequentially cleaned with acetone, ethanol and de-ionized water. The surfaces were then dried with lens cleaning paper and finally flushed with compressed air to remove remaining wastepaper or dust.

The top surface temperatures of the substrates were achieved through the temperature control of two heating cartridges, which were inserted into the substrates. The two heating cartridges were controlled by means of PID loops with an accuracy of $0.05^{\circ} \mathrm{C}$. Two thermocouple were respectively inserted into a hole $5 \mathrm{~mm}$ under each top surface of the substrate to monitor the temperatures.

The drops were injected and created through a 700 $\mu \mathrm{m}$ hole inside the substrate by a syringe pump system. The injection rate and volume could be regulated by a step motor, which was connected a step driver and PLC controller. In this experiments, the step motor controlled two syringes together, which ensured the two created drops in the two substrates be equal in volume.

The main goal of the experiments was measuring the contact angles in microgravity. In order to obtain clear images of the drops, a big background light and two CCD camera were set behind and before the drops, respectively. The images acquired by the CCD camera were transmitted to two video recorders for computer analysis after the experiments.

To analyze the data from obtained images automatically and accurately, a software program was introduced to determine some physical parameters of the drops, such as the contact angle, bottom diameter and volume. The software first adopted edge detection to find the true contour of the drops. Then, a Laplace equation, Newton-Raphson method and Rouge-Kutta method were introduced to approach the true contour (Rotenberg et al. 1983; Yu et al. 2009). The volume, surface area and contact angle of the drop were obtained

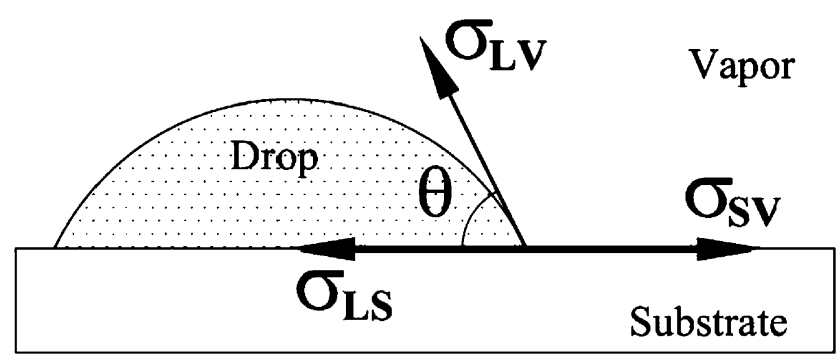

Fig. 2 Contact angle definition for a analyzed drop

based on the final calculated curve. The definition of contact angle is shown in Fig. 2, where $\sigma_{\mathrm{LV}}, \sigma_{\mathrm{LS}}$ and $\sigma_{\mathrm{SV}}$ are respectively the liquid-vapor, liquid-solid and solidvapor surface tensions. Note that the contact angle accuracy of the software could reach $\pm 1^{\circ}$ after calibrating with standard steel sphere. If we take the quality of the drop pictures obtained by the CCD and the symmetry of the drops into account, ordinarily, the accuracy could reach $\pm 3^{\circ}$.

Two liquids were used in this experiments, pure water and ethanol. The two liquids have different saturated vapor pressure, which is useful to study the influence of the evaporation effect. In addition, pure water is nonwettable for PTFE and aluminum, which means the contact angle is big enough to analyze easily. While for the ethanol, in despite of its low surface tension and wettable for aluminum, its high saturated vapor pressure is useful to supply reference for next satellite experiment.

\section{Results and Discussion}

The mechanisms of the evaporation drops are complicated. Making use of the above experimental apparatus and measurements techniques, several experiments have been carried out to study the contact angle behavior of evaporating drops in microgravity. There are many parameters to influence the contact angle. In this paper, we took the gravity level, surface temperature, status of the drop (sessile or pendant) and injection time (normal gravity or microgravity) into account separately to study the contact angle dependance on the above mentioned influence. Experimental conditions for all the experiments in present paper have been listed in Table 1.

G-jitter is an unavoidable problem in most microgravity experiments. The drop interface is very sensitive to the g-jitter, which has already resulted in some unclear pictures in our last parabolic experiment. Therefore, a good microgravity level is necessary to 
Table 1 Experimental conditions of all the experiments in present paper

\begin{tabular}{lllll}
\hline Status & Liquid & Surface & Temperature & Injecting \\
\hline Sessile & Water & Aluminum & Heating & $1 \mathrm{~g}$ \\
& & PTFE & Heating & $1 \mathrm{~g}$ \\
Pendant & \multirow{2}{*}{ Ethanol } & PTFE & Room & $1 \mathrm{~g}$ \\
& & Aluminum & Room & $1 \mathrm{~g}$ \\
& \multirow{2}{*}{ Water } & PTFE & Room & $1 \mathrm{~g}, \mu \mathrm{g}$ \\
\hline
\end{tabular}

perform accurate contact angle measurements. In the presented Drop Tower experiments, it was found that the microgravity level was better than that in parabolic campaigns except a small vibration when the drops just entering microgravity.

\section{Wettability}

As we know, a drop could display different wettability for different surface. Figure 3 shows the water drops on PTFE and aluminum during one experiment, i.e. the gravity level changes from normal to microgravity.

In this experiment, the drop was injected and created at normal gravity with a injection rate of $0.103 \mathrm{~mL}$ in $6 \mathrm{~s}$. Then, the drop was sustained to be steady for about $30 \mathrm{~s}$. The experiment began as soon as the drop tower capsule falling down, i.e. the overall experimental apparatus entered microgravity. As shown in Fig. 3, the contact angle of the drop decreases as soon as the gravity level reaching microgravity. For the drop on PTFE, the contact angle is around $110 \pm 5^{\circ}$ when the drop is created under terrestrial gravity. While in
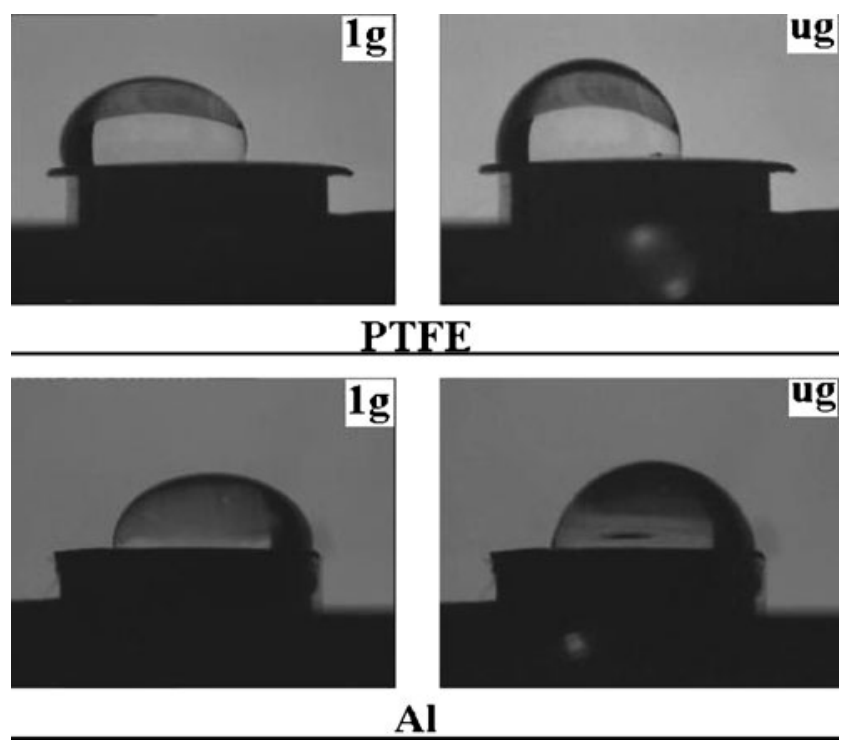

Fig. 3 Water drops on PTFE and Aluminum under different gravity level microgravity, the contact angle decreases $10^{\circ}$. The same for drop on aluminum, the contact angle has a sudden change from $90^{\circ}$ to $75^{\circ}$. At the same time, the drop has a evolution: the height of the drop increases and the bottom diameter of the drop decreases a little. Actually, in the study of drops, "bigger" drop (with high ratio of height to diameter) is usually easily to analyze, due to its easily identified contact angle. In this experiment, it is obvious that the contact angle on PTFE is always bigger than that on aluminum, which objectively reflects that for water the non-wettability on PTFE is better than aluminum. The same phenomenon was found for ethanol. And the mechanism of gravity level influence on the drop motion will be given after introducing contact angle dependence on temperature in the next section.

\section{Contact Angle Dependence on Temperature}

In above mentioned literatures, most of researchers focus on the time evolvement for a evaporating drop. They usually choose physical characteristic of liquids, roughness and chemical property of the surface and diameter of drops as the main parameters. However, there is rare study regarding the contact angle dependence on the surface temperature. In presented paper, we regulated the substrate temperature by means of heating cartridges and PID loops. Based on the approximately same room temperature $\left(30^{\circ} \mathrm{C}\right)$, the surface temperature was increased $+8^{\circ} \mathrm{C}, 15^{\circ} \mathrm{C}, 23^{\circ} \mathrm{C}$ and $30^{\circ} \mathrm{C}$ successively. Figures 4 and 5 show the variation of the contact angle on different surface temperatures for water drop on aluminum and PTFE, respectively.

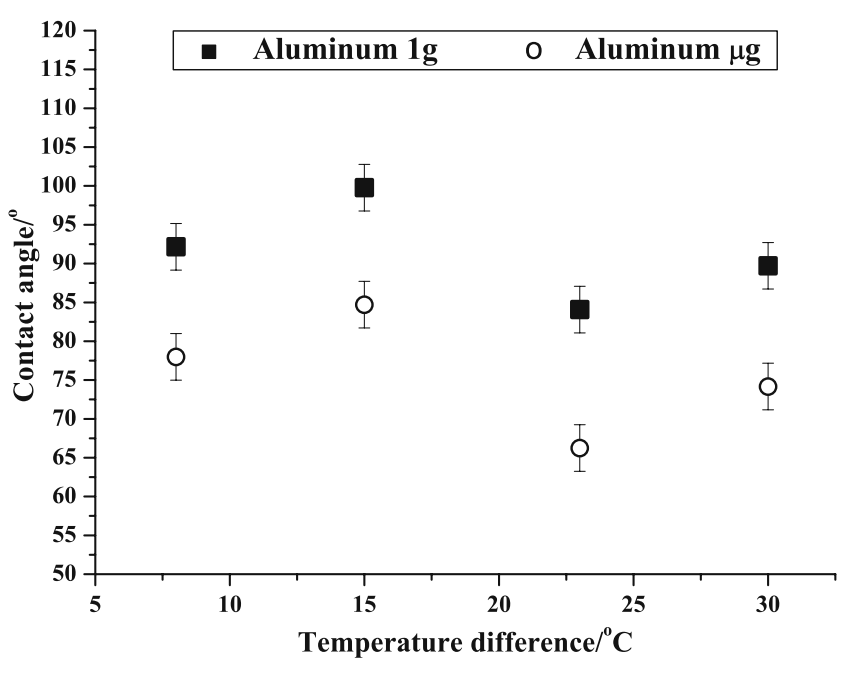

Fig. 4 Sessile water drops on aluminum: variation of contact angle for different surface temperatures 


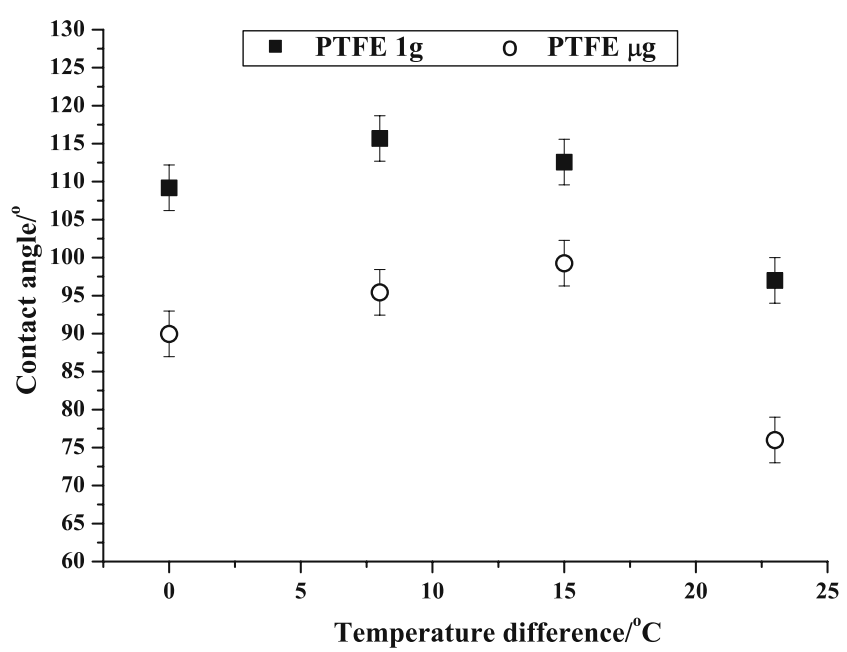

Fig. 5 Sessile water drops on PTFE: variation of contact angle for different surface temperatures

It could be seen that no matter the water drop on aluminum or on PTFE, the contact angles in normal gravity are always larger than that in microgravity. The schematic diagram of gravity level influence on the shape of a sessile drop is shown in Fig. 6. According to previous experimental studies, most drops are pinned at the surfaces for much of the lifetime of the drops, i.e. the vicinity of the drops are kept to be constant. The vicinity only removes inwards at the end of lifetime of the drops. Actually, the drops in presented experiments were also found to be pinning case. As shown in Fig. 6, gravity and surface tension influence the interface of the drop together when the drop is under terrestrial gravity. And the gravity effect "forces" the triple line to spread along the surface. While synchronously, the pinning vicinity of the drop "prevents" the trend of spreading. As a result, the two effects ultimately only "press" the liquid close to the vicinity downwards and outwards. As soon as the drop be in microgravity, the gravity influence vanishes. Therefore, the "pressed" liquid close to the vicinity rebounds to upper position, which decreases the contact angle, increases the height of the drop.

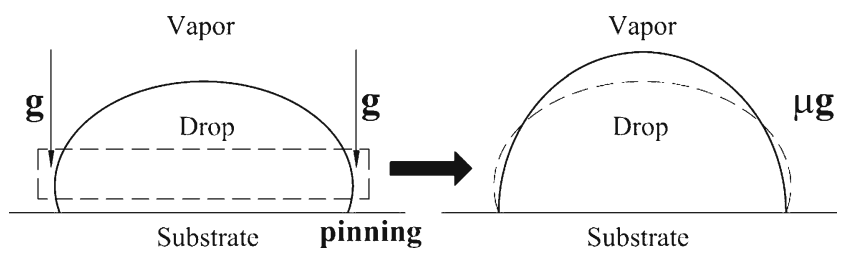

Fig. 6 Schematic diagram of gravity level influence on the shape of a sessile drop
Comparing the contact angle at different surface temperatures with each other, aluminum and PTFE display different behaviors. For aluminum, it seems that there is not evidently dependence for contact angle on the surface temperature, even when the drop is in microgravity. While for PTFE, it seems that there is a tendency: contact angle decreases as the temperature difference increasing. Actually, initial conditions, such as characteristic of the liquid, roughness and chemical property of the surface, play a dominate role to determine the shape of the drop. In present experiment, the good wettability for water on aluminum made the water drop pinning on the aluminum, which prevented the water drop changing with temperature. On the contrary, for the water drop on PTFE, worse wettability made it relatively easier for the drop to change with temperature. As we know, surface tension of water decreases with increasing temperature. Hereby, we can infer that contact angle of water drop decreases with increasing temperature difference taking account only of surface tension change with temperature.

\section{Contact Angle for Pendant Drop}

As above mentioned, convection in the drop was considered as an important influence on the heat transfer of the drop. For sessile drops, buoyancy and thermocapillary convection usually work together to influence the heat transfer. In order to approach the drops in future satellite experiments, pendant drops were introduced to perform similar experiments. At different moments during the presented experiment, the contour of an ethanol pendant drop changed evidently. The result is presented in Fig. 7.

Comparing the results on sessile drop, it could be seen that gravity effect plays opposite roles on sessile and pendant drops. Alike the description on how gravity influences the sessile drop, the gravity effect plays a more important role than surface tension and roughness of the surface to "pull" the pendant drop downwards. Once the pendant drop getting into microgravity, the disappearance of gravity effect leads to the "pulling" liquids upwards, which decreases the contact angle.

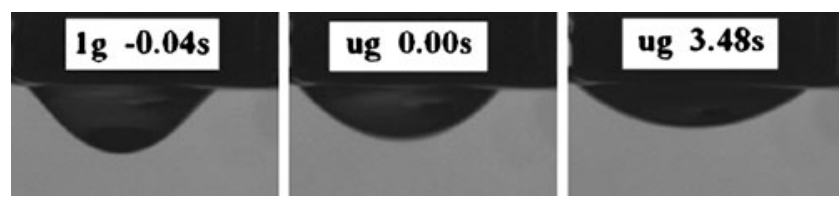

Fig. 7 Ethanol pendant drop on PTFE: variation of the drop contour at different moments during the experiment 
Former research found that the drop contact angle increased with the increasing drop diameter under terrestrial gravity. In presented paper, we chose ethanol to perform pendant drop experiment. The contact angles varying with drop diameters in microgravity is plotted on Fig. 8. We also plot the results of sessile drops obtained from a parabolic flight (Brutin et al. 2009) on the same figure for comparison. Note that the substrates and surfaces were same in the two experiments, while the drops were sessile water drops and pendant ethanol drops in parabolic and Drop Tower experiments, respectively. In parabolic experiments, we observed that the contact angles of sessile water drops in microgravity showed to be changeless with increasing drop diameter. Comparing the two results, although the pendant ethanol drops display smaller contact angles due to ethanol's lower surface tension, the same trend of changeless contact angles with varying drop diameters, could also be found in Drop Tower experiments. At the same time, it is found that the contact angles in parabolic experiments are more dispersed than that in Drop Tower experiments for the same range of drop diameter between 5.0 and $10.0 \mathrm{~mm}$, which is mainly introduced by the bigger g-jitter in parabolic campaigns.

\section{Contact Angle Change for Continuing Injection}

To study drop evaporation and validate the feasibility of creating drop in microgravity, it is necessary to create the drop without gravity. In Fig. 9, we present the continuing injection of a water pendant drop in

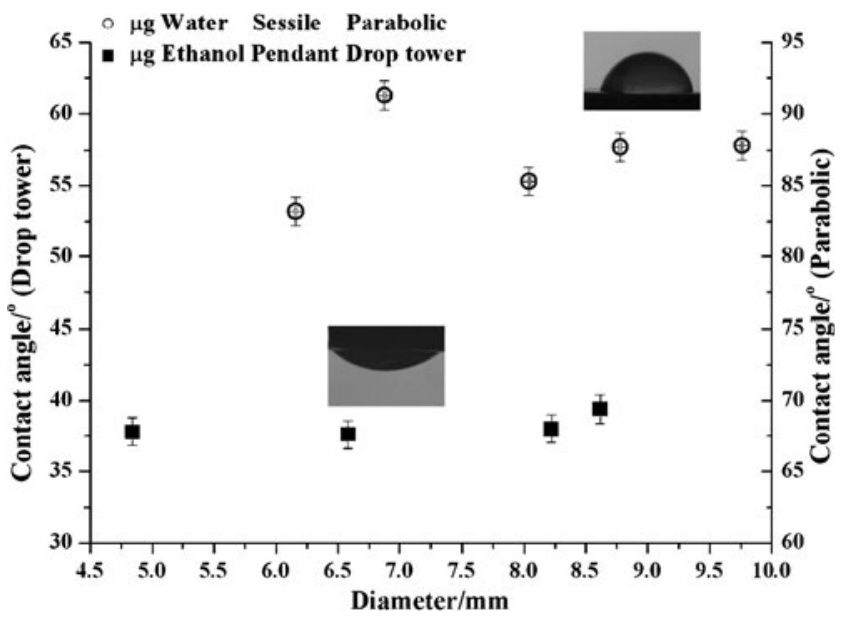

Fig. 8 Compare the result with that obtained from parabolic flight
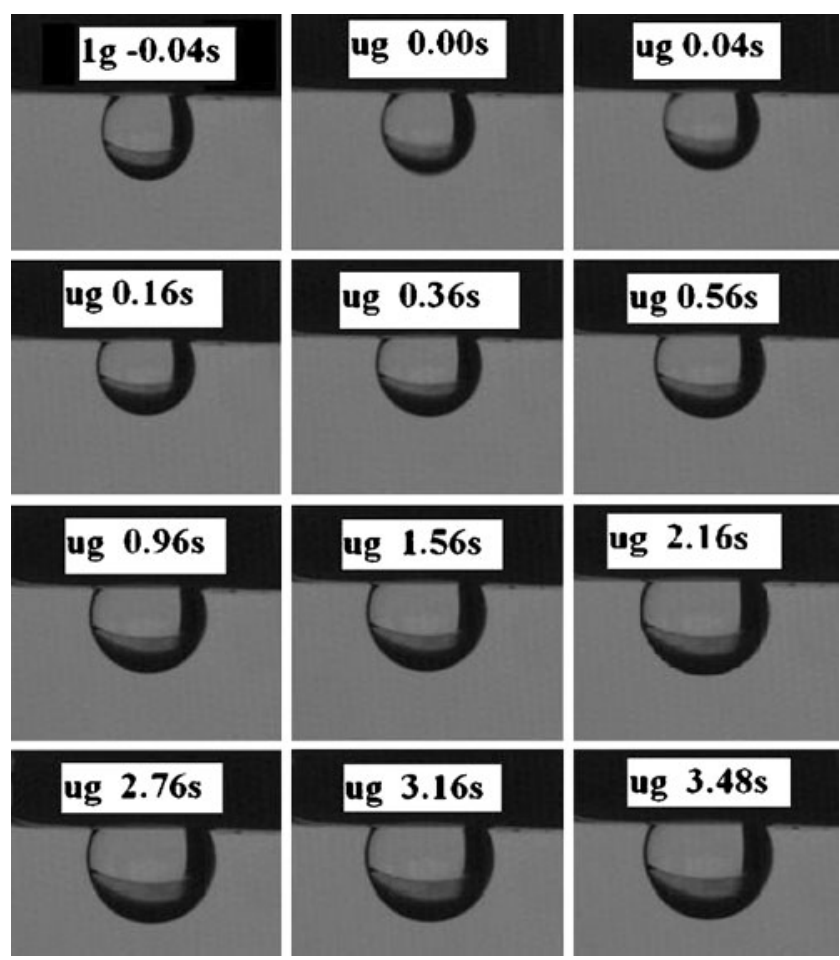

Fig. 9 Water pendant drop on PTFE: continuing creation of the drop in microgravity

microgravity with an injection flow rate of $13 \mu \mathrm{L} / \mathrm{s}$. In this experiment, we first injected to create a pendant drop in normal gravity. As soon as the drop getting into microgravity, the syringe pump continued to inject same volume of water to enlarge the drop in $2 \mathrm{~s}$ (smaller than the microgravity period of $3.6 \mathrm{~s}$ ). As shown in Fig. 9, the drop keeps constant after $2.16 \mathrm{~s}$.

During the time in microgravity, some typical points of the drop contact angle and bottom diameter for continuing injection are listed in Table 2. The syringe pump has a response time of $0.25 \mathrm{~s}$. As a result, for the contact angle, the drop changes the contour immediately with absence of gravity, exhibit a sudden change of contact

Table 2 Typical points on the drop contact angle and diameter during the continuing injection in microgravity

\begin{tabular}{lll}
\hline Time/s & Contact angle/degree & Diameter $/ \mathrm{mm}$ \\
\hline-0.04 & 112.0 & 2.47 \\
0.04 & 118.0 & 2.73 \\
0.24 & 119.7 & 3.02 \\
0.56 & 115.3 & 3.34 \\
1.56 & 113.2 & 3.60 \\
2.16 & 115.6 & 3.82 \\
2.76 & 114.4 & 3.84 \\
3.48 & 115.0 & 3.84 \\
\hline
\end{tabular}


angle and reach the steady status with a period of 0.25 $\mathrm{s}$. Then, the continuing injection begins, and the contact angle continues decreasing until the end of injection. It is mainly because that non-wettability of water on PTFE makes the pendant drop more easily to expand downwards than along the surface. After the injection finished, it takes about $0.25 \mathrm{~s}$ for the drop to achieve steady status. On the other hand, for the bottom diameter, the injection continues increasing the diameter. And the diameter stays to be steady after the injection. Note that this is just the primary investigation. More detailed analysis will be performed in the near future.

\section{Conclusion}

The behaviors of sessile and pendant drops in microgravity were studied experimentally by using the 3.6 $\mathrm{s}$ drop tower in Beijing. The wettability at different surfaces, contact angles dependance on the surface temperature, contact angle behavior in sessile and pendant drops and continuing injection were measured and analyzed.

A drop set on different surface (PTFE and aluminum) could exhibit different contact angles due to the wettability. According to the results on drop contact angle at different surface temperatures, for aluminum, it seems that there is not evidently dependence for contact angle on the surface temperature, even when the drop is in microgravity. While for PTFE, it seems that there is a tendency: contact angle decreases as the temperature difference increasing. Gravity effect plays opposite roles on determining the contour of sessile and pendant drops. Comparing the presented results with that obtained in a parabolic flight, they both display a trend that drop diameter has no obvious influence on drop contact angle in microgravity. With the goal of obtaining reference on future satellite experiments, a attempt of continuing injection was performed in microgravity, and the variation of the injected drop during the whole process has been observed clearly.

Based on former literature, the evaporation and convection of drops could influence evidently the heat transfer. Therefore, the next work is to investigate the drop evaporation and convection in a long time microgravity condition, especially in different thermal environments. So, our new experiments will be carried out to focus on the study regarding how evaporation influences the drops.
Acknowledgements This research was financially supported by the National Natural Science Foundation of China (Grant Nos. 10772185 and 50890182) and the Knowledge Innovation Project of Institute of Mechanics/CAS.

\section{References}

Akira, O.: Dynamic van derWaals theory of two-phase fluids in heat flow. Phys. Rev. Lett. 94, 054501-1-054501-4 (2005)

Bigioni, T.P., Lin, X.M., Nguyen, T.T., Corwin, E.I., Witten, T.A., Jaeger, H.M.: Kinetically driven self assembly of highly ordered nanoparticle monolayers. Nat. Mater. 5, 265-270 (2006)

Birdi, K.S., Vu, D.T., Winter, A.: A study o the evaporation rates of small water drops placed on a solid surface. J. Phys. Chem. 93, 3702 (1989)

Bourges-Monnier, C., Shanahan, M.E.R.: Influence of evaporation on contact angle. Langmuir 11, 2820-2829 (1995)

Brutin, D., Zhu, Z.Q., Rahli, O., Xie, J.C., Liu, Q.S., Tadrist, L.: Sessile drop in microgravity: creation, contact angle and interface. Microgravity Sci. Technol. 21(Suppl 1), S67-S76 (2009)

Crafton, E.F., Black, W.Z.: Heat transfer and evaporation rates of small liquid droplets on heated horizontal surfaces. Int. J. Heat Mass Transfer 47, 1187-1200 (2003)

Grandas, L.: Evaporation d'une goutte sessile: etude experimentale des transferts de chaleur et de masse. Ph.D. thesis, Universite de Provence (2004)

Grandas, L., Reynard, C., Santini, R., Tadrist, L.: Experimental study of the evaporation of a sessile drop on a heat wall. Int. J. Therm. Sci. 44(2), 137-146 (2005)

Law, C.K.: Recent advances in droplet vaporization and combustion. Pror. Energy Combust. Sci. 8, 171 (1982)

Panwar, A.K., Barthwal, S.K., Ray, S.: Effect of evaporation on the contact angle of a sessile drop on solid substrates. J. Adhes. Sci. Technol. 17(10), 1321-1329 (2003)

Picknett, R.G., Bexon, R.: Evaporation of sessile or pendant drops in still air. J. Colloid Interface Sci. 61, 336 (1977)

Ponter, A.B., Boyes, A.P.: The relation between contact angle and drop size for water at its boiling point for a pressure range 50-760 Torr. Can. J. Chem. 50, 2419 (1972)

Rotenberg, Y., Boruvka, L., Neumann, A.W.: Determination of surface tension and contact angle from the axisymmetric fluid interfaces. J. Colloid Interface Sci. 93, 169-183 (1983)

Ruiz, O.E., Black, W.Z.: Evaporation of water droplets placed on a heated horizontal surface. J. Heat Transfer 124, 854-863 (2002)

Shahidzadeh-Bonn, N., Rafai, S., Azouni, A., Bonn, D.: Evaporating droplets. J. Fluid Mech. 549, 307-313 (2006)

Sirignano, W.A.: Fuel droplet vaporization and spray combustion theory. Pror. Energy Combust. Sci. 9, 291 (1983)

Takata, Y., Hidaka, S., Cao, J.M., Nakamura, T., Yamamoto, H., Masuda, M., Ito, T.: Effect of surface wettability on boiling and evaporation. Energy (Oxf.) 30(2-4), 209-220 (2005)

Savino, R., Paterna, D., Favaloro, N.: Buoyancy and marangoni effects in an evaporating drop. J. Thermophys. Heat Transf. 16(4), 562-574 (2002)

Yu, Q., Cai, S.J., Zhu, Z.Q., Liu, Q.S., Zhou, B.H.: Droplet image feedback control system in evaporation experiment. Microgravity Sci. Technol. 22(2), 139-144 (2010) 